\title{
Development of Weather-Based Predictive Models for Fusarium Head Blight and Deoxynivalenol Accumulation for Spring Malting Barley
}

K. D. Bondalapati, Plant Science Department, South Dakota State University, Brookings, US-SD 57007; J. M. Stein, Plant Science Department, SDSU; S. M. Neate, Department of Plant Pathology, North Dakota State University, Fargo, US-ND 58102; S. H. Halley, North Dakota State University Langdon Research Extension Center, Langdon, US-ND 58249; L. E. Osborne, Plant Science Department, SDSU; and C. R. Hollingsworth, University of Minnesota Research and Outreach Center, Crookston, US-MN 56716

\begin{abstract}
Bondalapati, K. D., Stein, J. M., Neate, S. M., Halley, S. H., Osborne, L. E., and Hollingsworth, C. R. 2012. Development of weather-based predictive models for Fusarium head blight and deoxynivalenol accumulation for spring malting barley. Plant Dis. 96:673-680.

The associations between Fusarium head blight (FHB), caused by Gibberella zeae, and deoxynivalenol (DON) accumulation in spring malting barley (Hordeum vulgare) and hourly weather conditions predictive of DON accumulation were examined using data from six growing seasons in the U.S. Northern Great Plains. Three commonly grown cultivars were planted throughout the region, and FHB disease and DON concentration were recorded. Nine predictor variables were calculated using hourly temperature and relative humidity during the 10 days preceding full head spike emergence. Simple logistic regression models were developed using these predictor variables based

on a binary threshold for DON of $0.5 \mathrm{mg} / \mathrm{kg}$. Four of the nine models had sensitivity greater than $80 \%$, and specificity of these models ranged from 67 to $84 \%(n=150)$. The most useful predictor was the joint effect of average hourly temperature and a weighted duration of uninterrupted hours (h) with relative humidity greater than or equal to $90 \%$. The results of this study confirm that FHB incidence is significantly associated with DON accumulation in the grain and that weather conditions prior to full head emergence could be used to accurately predict the risk of economically significant DON accumulation for spring malting barley.
\end{abstract}

Fusarium head blight (FHB), caused by the fungus Gibberella zeae (Schwein) Petch (anamorph: Fusarium graminearum Schwabe), continues to be a serious problem for barley (Hordeum vulgare L.) producers in the U.S. Northern Great Plains and elsewhere. Economic losses associated with FHB occur because of the blighting of florets (reduction in grain number), disruption of grain fill (shriveled kernels leading to lower test weight), and most importantly through the contamination of grain with trichothecene mycotoxins, primarily deoxynivalenol (DON). Tolerances for DON in malting barley are generally lower than those for food or feedgrade barley because of the associations between DON, G. zeaeinfected kernels, and gushing in beer (15). Gushing is the spontaneously over-foaming of beer in the absence of agitation (16) and has been found to be the result of fungal hydrophobins, and possibly other proteins, being present at elevated levels in the malt $(15,34)$. DON concentration in grain is used to estimate this risk at the elevator, and severe discounts or even crop rejection can occur if the level detected exceeds $0.5 \mathrm{mg} / \mathrm{kg}$.

Resistance to FHB is currently inadequate in industry-approved malting barley cultivars, and management is limited to reducing infield inoculum and/or the application of fungicide after spike emergence. Inoculum management through crop rotation and/or residue management (e.g., tillage) has been postulated to reduce both the number of $G$. zeae propagules reaching the spikes and the final DON concentration in wheat $(11,36)$; however, this approach is not completely effective since the spores of $G$. zeae can become air-

\section{Corresponding author: J. M Stein, E-mail: jeffrey.stein@monsanto.com}

Current address of J. M. Stein: Monsanto Company, Waterman, US-IL 60556. Current address of S. M. Neate: Leslie Research Centre, Toowoomba, AU-QLD 4350. Current address of L. E. Osborne: Pioneer HiBred International, Brookings, US-SD 57006. Current address of C. R. Hollingsworth: USDA-APHIS-CHPST, Raleigh, US-NC 27606.

Accepted for publication 1 November 2011.

http://dx.doi.org/10.1094/PDIS-05-11-0389

(C) 2012 The American Phytopathological Society borne and travel moderate distances $(22,35)$. The application of certain fungicides after spike emergence but prior to infection can significantly reduce both FHB and DON accumulation in the grain (41). Application timing is critical, and therefore a risk advisory system could help growers make informed management decisions and reduce the risk of economic loss from FHB.

Weather based prediction models have been developed for FHB of wheat in Argentina (26), Belgium (10), Italy (32), and the United States (7-9). De Wolf et al. (9) used 50 location-years from Ohio, North Dakota, Missouri, and Kansas to create logistic regression models to predict the probability of an FHB epidemic as defined by a field severity of $\geq 10 \%$. The authors reported a strong association between FHB severity and extended periods of high relative humidity $(\geq 90 \%)$ with temperature between 15 and $30^{\circ} \mathrm{C}$ during the period of anthesis (9). Later generation wheat scab models included only mean relative humidity during the preflowering period (7). Similar parameters were reported as being important for FHB prediction in Argentina (26) and under controlled-environment conditions (1). Risk prediction in Italy was based on calculations of sporulation, dispersal, and infection rates (INF) while considering host growth stage. These rates were influenced by different weather conditions such as air temperature, relative humidity, and rain intensity (32). In Belgium, FHB risk in winter wheat was assessed using a combination of agricultural factors (previous crop, cultivar, etc.) and cumulated leaf wetness duration (h) with temperature $>12^{\circ} \mathrm{C}$ during 7 days before and after the flowering date (10). In addition, quantitative prediction models were developed to predict DON concentration in wheat using weather variables in Canada (19), Czech Republic (20,39), and the Netherlands (38). Hooker et. al. (19) reported that DON concentration was significantly influenced by rainfall and temperature at three critical time periods during pre- and postheading. In the Netherlands, separate linear regression models were developed to predict DON concentration for growers and food safety authorities separately (38). The model developed for growers used weather information for 17 days preceding and 10 days after the flowering date, whereas the model for industry included weather information from flowering to harvest. Both models considered agronomic information such as field location and level of cultivar resistance, etc. Average hourly 
temperature, sum of hourly rainfall, and duration of hours with relative humidity $>80 \%$ were determined as significant variables to predict DON in both models (38). The neural network model developed in the Czech Republic to predict DON concentration included preceding crop and weather-based variables such as average temperature and precipitation in April, and weather for the 5 days preceding anthesis $(20,39)$. With few exceptions, all of the models noted above rely on variables that include some measurement of temperature and humidity/wetness.

Due to the large potential economic impact from FHB in the U.S. malting barley crop, growers in high-risk regions such as the Northern Great Plains could benefit greatly from an advisory system that supports the fungicide application decision process. To address this need, a project was initiated in 2005 to: (i) determine how DON concentration was correlated with disease in barley, (ii) develop an FHB infection model based on temperature and wetness duration, and (iii) develop risk models that were predictive of DON levels greater than $0.5 \mathrm{mg} / \mathrm{kg}$.

\section{Materials and Methods}

Field data collection. Experiments were conducted using a set of regionally adapted malting barley cultivars grown at multiple locations in North Dakota and South Dakota (2005-2010), and Minnesota (2005, 2007-2009) or Montana (2006). Data from the years 2005-2009 were used to develop potential predictive models, and data from the year 2010 was used for validation. At least three cultivars, namely 'Conlon' (2-row) and 'Robust' and 'Tradition' (both 6-row), were common at all locations. These were chosen because they were adapted to the region, were commonly grown, and were on the American Malting Barley Association recommended list in 2005 (2). Plots were sown with a small-plot planter, a minimum of $1.5 \times 4.6 \mathrm{~m}$ in size, replicated four times in a randomized complete block design (RCBD), and maintained using standard agronomic practices for the region. The plots were not inoculated, nor were they manipulated in any way to increase the probability of infection.

Crop growth was monitored regularly throughout the growing season, and the date at which each cultivar was at Zadoks stage 59 (43) (spikes on main stems fully emerged) was noted; hereafter this is referred to as the heading day. When the plants in each replicate plot were at the soft-dough stage (approximately 18 to 21 days after heading or at Zadoks growth stage 85), a minimum of 25 spikes per plot ( $5+$ bundles of five) were rated for FHB incidence and field severity, with the latter being calculated as the number of symptomatic spikelets divided by total number of spikelets per spike. Plots were harvested at the end of the growing season using a small-plot combine, and $50 \mathrm{~g}$ of grain from each plot was submitted for mycotoxin analysis. In all cases, the mean DON concentration for all replicate plots of a single cultivar at each location-year was used in the analyses. Pearson's correlation coefficients were calculated between incidence, field severity, and DON concentration to evaluate associations.

In addition to disease and mycotoxin data, nearby weather stations were used to record hourly environmental conditions. At each location, the weather station was typically within $35 \mathrm{~m}$ of the research plots. For the locations in South Dakota and Minnesota/ Montana, an Onset HOBO Micro Station Data logger (Onset Computer Corporation, Bourne, MA) was used to record the weather data on an hourly basis. For the locations in North Dakota, hourly weather data were downloaded from the ND automated weather network. At a minimum, collected weather data include temperature, relative humidity (RH), and precipitation (incidence and rate). These factors were used to calculate other variables (e.g., average hourly temperature). Hourly weather observations were collected for at least 9 days preceding, and including, the heading day for each cultivar at all location-years.

As noted previously, one of the objectives of this project was to develop a model that was predictive of the accumulation of economically significant DON concentration in barley grain. The concentration of $0.5 \mathrm{mg} / \mathrm{kg}$ was used for this purpose for the afore- mentioned reasons. A binary response variable, eDON (e conomic DON), was created based on whether the mean concentration for each cultivar at each location-year met or exceeded this threshold, i.e., those cultivars with less than $0.5 \mathrm{mg} / \mathrm{kg}$ DON were assigned a value of 0 , whereas those with greater than or equal to this concentration were assigned a value of 1 . For example, if the mean DON concentration for a cultivar-location-year was $0.7 \mathrm{mg} / \mathrm{kg}$, it would be assigned an eDON value of 1 .

Development of an infection model for FHB in a controlled environment. Although not always strong, significant positive correlations between FHB symptoms and DON concentration were reported in malting barley from fields in Minnesota, North Dakota, and South Dakota over the years 1993-1996 (33), in Eastern Canada (4), and in wheat over multiple studies (28). Based on these associations, an infection model was developed for FHB, which was further extended to predict the risk of a DON concentration greater than or equal to $0.5 \mathrm{mg} / \mathrm{kg}$.

The infection model incorporated the nonlinear relationship between temperature $(t)$ and the duration of continuous wetness $(w)$ on resulting symptom development. This relationship was expressed using the modified form of the Weibull function given by Duthie (12):

$Y=f(w, t)=A\left(1-\exp \left\{-[B(w-C)]^{D}\right\}\right)$

in which $B$ varies with temperature according to

$$
B=f(t)=E[(H+1) / H] H^{1 /(H+1)}\{\exp [(t-F) G /(H+1)]\} /\{1+\exp [(t-F) G]\}
$$

$Y$ is a measure of the response in terms of symptom development at any given $w$ and $t$. The typographical error in the equation 3.2 of Duthie (12) was noticed by Arauz et al. (3), and we used the corrected equation. The description of each parameter is clearly explained by Duthie (12), but can be summarized as follows. The parameter $A(0<A<1)$ characterizes the upper limit on the response, the parameter $B(0<B<1)$ characterizes the intrinsic rate of increase in the response with respect to $w$ (considered as a function of $t), C(>0)$ is the lag period of $w$ before the response begins, and the parameter $D(>0)$ represents the period of wetness in which the response decelerates. The parameter $E(>0)$ characterizes the scale of response to $t, F$ is proportional to the optimal $t, G(>0)$ represents intrinsic rate of change in the response with respect to $t$, and $H(>0)$ characterizes asymmetry in the response to $t$.

To estimate the parameters in equations 1 and 2, disease data from a controlled environment study for barley were required, but not available, and therefore data from Anderson's study of FHB infection in wheat (1) were used. In short, the proportion of symptomatic wheat spikelets at a given $t$ and $w$ was considered as the response variable. To reduce over parameterization, the parameters $A, C$, and $F$ were fixed to the values of $1.0,12.0$, and 25.0 , respectively, under the assumptions of: (i) all spikelets could become blighted (i.e., 100\%), (ii) no infection occurred in the study before $12 \mathrm{~h}$ of continuous wetness, and (iii) the proportion of blighted spikelets reached a maximum rate with respect to wetness at $t=25^{\circ} \mathrm{C}$ in Anderson's data (1). Best-fit parameter estimates for $D, E, G$, and $H$ were obtained using the Marquardt iterative method of the NLIN procedure in SAS (Statistical Analysis System ver. 9.1; SAS Institute, Cary, NC). Linear regression of the observed against predicted proportions of blighted spikelets was conducted and analyzed by testing the significance of the difference in the intercept from 0 and the slope from 1 (14).

Extension of the infection model to the field. Since environment is not constant in the field, infection can occur over several days, and inoculum does not reach the plant in a single dose, several input variables were developed to estimate the temperature $(t)$ and wetness duration $(w)$ in the above model. All were calculated over the 10-day interval as noted above. For temperature, these included average hourly temperature (AvgT), average daily minimum temperature (AvgMinT), and average daily maximum temperature (AvgMaxT). The input variables for wetness duration 
were based on the hours with $\mathrm{RH} \geq 90 \%$ under the assumption that free moisture was present on the plants (37). These included the number of hours with $\mathrm{RH} \geq 90 \%$ (RH90), single longest uninterrupted duration of hours with $\mathrm{RH} \geq 90 \%$ (drRH90), and a weighted duration of hours with $\mathrm{RH} \geq 90 \%$ (wRH90). wRH90 prioritized longer uninterrupted wetness durations in a manner similar to a degree-day in that a minimum duration of $8 \mathrm{~h}$ was required to increase the weight for a given uninterrupted humidity run $(\mathrm{RH} \geq 90 \%)$ during the 10-day interval. This was calculated using the formula:

$\mathrm{wRH} 90=\sum_{i} x_{i}\left[1+\frac{W_{i}}{\sum_{i} W_{i}}\right] ; \quad W_{i}=\left\{\begin{array}{l}x_{i}-8 \text { if } x_{i}>8 ; \\ 0 \text { otherwise }\end{array}\right.$

where $x_{i}$ is an instance of uninterrupted wetness duration (h) when $\mathrm{RH} \geq 90 \%$ and $i$ is an indicator to represent such uninterrupted durations in the 10-day interval. An example calculation of wRH90 for a specific event during the 10-day interval can be found in Table 1 . The maximum $x_{i}$ possible was $240 \mathrm{~h}$. Moreover, the weighted duration of hours with $\mathrm{RH} \geq 90 \%$ was always greater than or equal to the duration of hours with $\mathrm{RH} \geq 90 \%$ (wRH90 $\geq$ RH90). Although there are other ways to estimate wetness durations in the field that may be more representative of dew periods $(17,31)$, they were not used due to the unavailability of environmental variables used in these measurements, such as wind speed.

For each event, $Y$ was calculated using equation 1 by replacing $t$ with AvgT, AvgMinT, or AvgMaxT and by replacing $w$ with RH90, drRH90, or wRH90. A total of nine variables were calculated from equation 1 using the aforementioned alternatives for $t$ and $w$, hereafter referred as Weibull variables. The parameters $A$ and $F$ were fixed at 1.0 and $25^{\circ} \mathrm{C}$ for the reasons given above. However, parameter $C$ was fixed at $0 \mathrm{~h}$ instead of $12 \mathrm{~h}$ for two reasons: (i) equation 1 was undefined when RH90 was less than 12 h, and (ii) FHB infection can possibly occur in the field even if there were less than $12 \mathrm{~h}$ of $\mathrm{RH} \geq 90 \%$ during the 10-day interval, since there might be many short, but conducive, periods. Estimates from the fitting of the model to Anderson's data were used for the remaining parameters $D, E, G$, and $H$.

Pearson's correlation coefficients between disease metrics (incidence, field severity, DON, and $\mathrm{eDON}$ ) and the nine Weibull variables were calculated to assess the association between disease metrics and variables obtained using weather conditions in the field.

Development of logistic regression models for eDON. Since three cultivars were planted at each location, the significance of the cultivar effect on eDON accumulation was tested using a chisquare test. The null hypothesis was that the three had an equal proportion of positive eDON events $(\mathrm{eDON}=1)$. Cultivar was considered as a predictor for $\mathrm{eDON}$ if there was enough evidence to show that the proportion of positive eDON events was significantly different among cultivars.

The relationship between the binary eDON and the nine Weibull variables was evaluated using logistic regression. The function 'Irm' (Logistic Regression Model) in the Design package from R $(18,30)$ was used to perform the analysis. Classification of events into positive or negative for eDON requires an arbitrary threshold probability, often set at 0.5 . However, the selection of the threshold probability $(p)$ influences model accuracy, sensitivity, and specificity. Accuracy refers to the proportion of events that are classified correctly, sensitivity (or true positive proportion, TPP) refers to the proportion of positive DON events that are classified correctly, and specificity (or true negative proportion, TNP) refers to the proportion of negative DON events that are classified correctly. ROC curves were created for each model as a plot of sensitivity (TPP) versus 1-specificity (false positive proportion, FPP) by allowing the threshold probability to vary over the range of possible values from 0 to $1(21,24)$. The area under the ROC curve (AUROC) and its standard error was calculated for each curve using the software MedCalc (MedCalc Software, Mariakerke, Belgium). A standard normal Z-statistic and associated $p$ value were calculated to test the null hypothesis that AUROC $>$ 0.5 .

For each model, the probability of a positive eDON $\left(p^{*}\right)$ was determined by selecting the $p$ that maximizes the Youden's index (42). Youden's index is calculated by $J=$ Sensitivity + Specificity- 1 and represents the point on the ROC curve that is at the greatest vertical difference between the ROC and the noninformative curve. Sensitivity represents the proportion of positive DON events that are classified correctly, and specificity represents the proportion of negative DON events that are classified correctly. Model accuracy, sensitivity, and specificity for each model were calculated using the cut-off threshold obtained from Youden's index.

Model validation. Accuracy of the logistic regression models was evaluated using a validation data set of 29 events $(n=29)$ These events were collected from 10 research fields from South Dakota and North Dakota in 2010 and were independent of the events that were used to develop the regression models. The same cultivars were used as in the model development phase. Weather and disease data at these events were obtained in an identical fashion as described earlier. An event was observed as positive eDON when the DON concentration was greater than or equal to 0.5 $\mathrm{mg} / \mathrm{kg}$ and predicted as positive eDON when the probability of being a positive eDON event was greater than or equal to $p^{*}$. The prediction accuracies (total accuracy, sensitivity, and specificity) were computed by comparing the observed and predicted responses.

Derivation of optimal decision threshold. The cut-off threshold $\left(p^{*}\right)$ based on the Youden's index assumes equal weight to both

Table 1. Example calculation of the weighted duration of hours with RH $\geq 90 \%$ (wRH90)

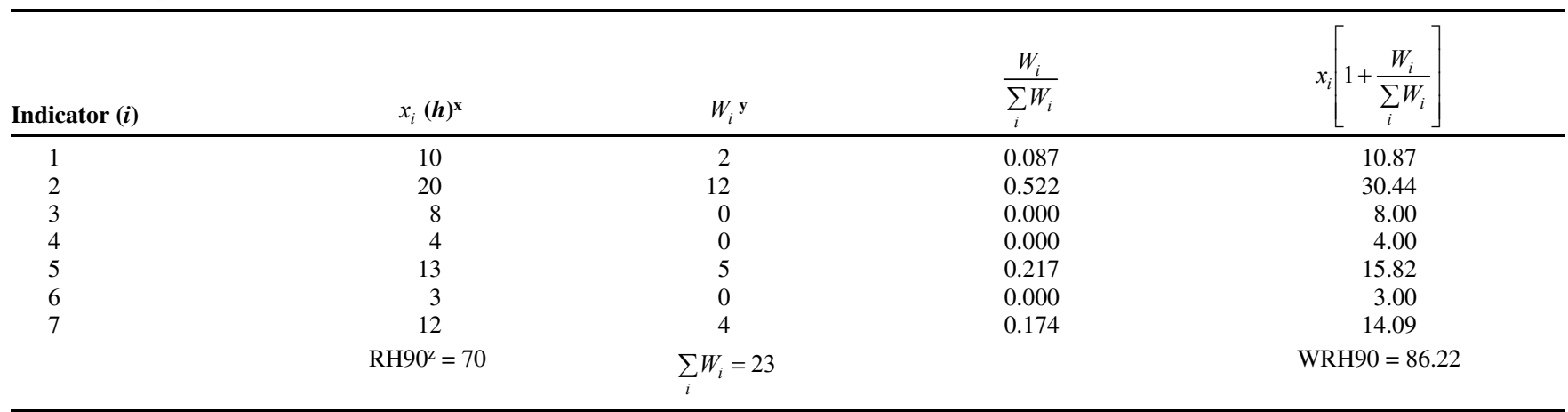

\footnotetext{
${ }^{x}$ Durations of uninterrupted hours with $\mathrm{RH} \geq 90 \%$ during the 10-day interval before and including the heading day for a specific event.

${ }^{\mathrm{y}} W_{i}=\left\{\begin{array}{l}x_{i}-8, \text { if } x_{i}>8 \\ 0 \text { otherwise. }\end{array}\right.$

${ }^{\mathrm{z}}$ RH90: Total duration of hours with $\mathrm{RH} \geq 90 \%$ during the 10-day interval.
} 
sensitivity and specificity. Economically, sensitivity and specificity are often not of equal importance in disease management, as the unnecessary application of a fungicide is usually less costly than crop losses due to severe disease. Fabre et al. (13) discussed the calculation of optimal decision threshold $\left(p_{T}\right)$ in terms of a cost function, which minimizes average management costs. Using $p$ as the probability of a positive DON event calculated from the logistic regression model, then the definition of a decision threshold, is a value $p_{T}$ such that if $p \geq p_{T}$ the event is classified as positive DON event which recommends a fungicide spray and if $p<p_{T}$ the event is classified as negative DON event which recommends no fungicide spray. Following the similar notation used by Fabre et al. (13), let $\mathrm{D}^{+}$denote the observed positive DON event and $\mathrm{T}^{+}$denote the predicted positive DON event. Conversely, $\mathrm{D}^{-}$and $\mathrm{T}^{-}$represent the observed and predicted negative DON events, respectively. Prev represents the a priori probability that DON concentration will be greater than the threshold value of $0.5 \mathrm{mg} / \mathrm{kg}$. The cost associated with $\mathrm{T}$ and $\mathrm{D}$ were represented by $C^{++}$(true positive), $C^{+-}$(false positive), $C^{-+}$(false negative), and $C^{--}$(true negative). The expected cost associated with $p_{T}$ for a given level of Prev is

$$
\begin{aligned}
& C\left(p_{T}\right)=\operatorname{Pr} e v \times\left[S e \times C^{++}+(1-S e) \times C^{-+}\right]+(1-\operatorname{Pr} e v) \times \\
& {\left[S p \times C^{--}+(1-S p) \times C^{+-}\right]}
\end{aligned}
$$

where $S e$ represents sensitivity and $S p$ represents specificity $(13,24)$. The expected cost given in equation 4 considers both model efficiency ( $\mathrm{Se}$ and $\mathrm{Sp}$ ) as well as disease prevalence (Prev). McRoberts et al. (23) discussed a new framework for decision making theory by focusing on probability of disease and cost of decision errors. The authors simplified equation 4 by assuming the cost of true positives and true negatives as zero and modified the cost equation in terms of the ratio of costs for false positives and false negatives.

Economic losses in a malt barley crop from FHB occur both in terms of yield and quality reductions, making estimating typical losses difficult. For the purposes of this study, losses were based on statewide yield averages for North Dakota in 2010 (180.5 bu/ha) and commodity prices for malt $(\$ 3.70 / \mathrm{bu})$ versus feed $(\$ 2.05 / \mathrm{bu})$ barley in August 2010 (USDA-NASS). The reduction in yield for an unsprayed crop was found to be $98 \%$ of the treated crop during an FHB epidemic, based on data generated from a separate study (data not shown). It was also assumed that the occurrence of FHB in an untreated crop would result in the grain failing to meet malt standards, and thus being sold for a reduced amount per bushel (\$1.65 less). Fungicide application was assumed to be $\$ 50$ per hectare based on typical product plus applicator costs. In short, average loss associated with a false negative $\left(C^{-+}\right)$event was estimated to be $\$ 257$ per hectare. Both $C^{++}$and $C^{+-}$were considered as the average cost associated with the fungicide spray, and the cost of a true negative $\left(C^{--}\right)$was considered $\$ 0$ per hectare.

The optimal decision threshold $\left(p_{T}\right)$ was considered as the value that minimizes the $C\left(p_{T}\right)$ given in equation 4 for a given level of Prev. This can be identified by setting zero to the first derivative of $C\left(p_{T}\right)$ with respect to $p_{T}$ as explained by Fabre et al. (13). To obtain the analytical approximation of first derivative of $S e(\Delta S e)$ and first derivative of $S p(\Delta S p), S e$ and $S p$ were estimated by fitting the following models to the observed data:

$$
\begin{aligned}
& \operatorname{Se}\left(p_{T}\right)=1-k e \times \exp \left(r e \times p_{T}-1\right) \\
& \operatorname{Sp}\left(p_{T}\right)=r p \times \ln \left(k p \times p_{T}+1\right)
\end{aligned}
$$

The parameter estimates for $k e, r e, k p$, and $r p$ were estimated by using the NLIN procedure in SAS. The optimal decision threshold $\left(p_{T}\right)$ for a range of values of Prev is identified by minimizing:

$$
\left[\Delta S p \times(1-\operatorname{Pr} e v) \times\left(C^{--}-C^{+-}\right)+\Delta S e \times \operatorname{Pr} e v \times\left(C^{++}-C^{-+}\right)\right]^{2}
$$

The Solver application in Microsoft Excel (Version 12.1.1, Microsoft, Redmond, WA) was used to obtain $p_{T}$ for a given range of Prev.

\section{Results}

Successful field studies were conducted at a total of 51 locationyears with three cultivars common at each location-year $(n=150)$. The DON data were missing for three events at different locations. Approximately 23\% (34 out of 150) of the events had DON accumulation of $\geq 0.5 \mathrm{mg} / \mathrm{kg}$. FHB incidence was significantly correlated with DON concentration $(r=0.45, P<0.001)$. A lower but significant positive correlation was observed between field severity and DON concentration $(r=0.30, P<0.001)$.

Development of an infection model for FHB. Estimates along with the $95 \%$ confidence interval for parameters $D, E, G$, and $H$ of equations 1 and 2 are presented in Table 2. Parameters had low standard errors and narrow confidence intervals, which did not include 0 . The $R^{2}$ for the linear regression model between observed and predicted proportion of spikelets blighted based on Anderson's wheat FHB data was 0.92 . The $F$ test failed to reject the null hypotheses of intercept equal to $0(P=0.76)$ and slope equal to $1(P$ $=0.52$ ). The response generated by equation 1 using the parameter estimates from Table 2 reached maximum at $t=25^{\circ} \mathrm{C}$ under prolonged wetness $(w>70 \mathrm{~h})$ and decreased when the temperature increased or decreased from the optimum even when $w=80 \mathrm{~h}$ (Fig. 1). The temperature response was asymmetrical, with slightly higher disease incidence when the temperature was cooler than the optima than when warmer.

Extending the infection model to the field environment. Nine variables were developed from the Weibull function given in equation 1 in which $t$ and $w$ were replaced with different measurements of temperature and duration of wetness in the field. The correlation coefficients with disease metrics (incidence, field severity, DON, and eDON) are given in Table 3. All nine were significantly and positively correlated to FHB incidence, field severity, DON, and eDON (Table 3 ). The variable $\mathrm{Wb}_{5}$ had the highest association with eDON, followed by $\mathrm{Wb}_{1}$.

Logistic regression models to predict eDON. The 6-row cultivars Robust and Tradition tended to have numerically higher DON concentrations as well as a larger number of eDON events than the 2-row cultivar Conlon (data not shown); however, there was insufficient evidence to reject the null hypothesis that the proportion of eDON events were equal for the three cultivars $(P=0.61)$ and therefore cultivar was not considered as a predictor for eDON.

Since the nine Weibull variables had significant correlation with eDON $(0.35 \leq r \leq 0.63)$ (Table 3$)$, simple logistic regression models were developed to predict eDON (Table 4). Total prediction accuracies, sensitivities, specificities, and AUROCs of all models

Table 2. Parameter estimates and associated statistics for the Weibull infection model using disease incidence data from a controlled environment study with wheat

\begin{tabular}{lcccc}
\hline & & & \multicolumn{2}{c}{ 95\% Confidence interval } \\
\cline { 3 - 4 } Parameter $^{\mathbf{z}}$ & Estimate & Asymptotic standard error & Lower & Upper \\
\hline $\mathrm{D}$ & 2.6475 & 0.0903 & 0.8859 & 1.2628 \\
$\mathrm{E}$ & 0.0252 & 0.0015 & 0.0222 & 0.0282 \\
$\mathrm{G}$ & 0.4804 & 0.4354 & 1.7393 & 3.5558 \\
$\mathrm{H}$ & 1.0744 & 0.0436 & 0.3895 & 0.5713 \\
\hline
\end{tabular}

${ }^{\mathrm{z}}$ Parameter estimates were obtained by using the Marquardt procedure defined in PROC NLIN in SAS. Response variable was considered as proportion of inoculated wheat spikelets blighted at different temperatures and exposure period to wetness provided by Andersen (1). Parameters A, C, and F were fixed at $1,12 \mathrm{~h}$, and $25^{\circ} \mathrm{C}$ as explained previously. 
for both training and validation data sets are presented in Table 4. These logistic regression models are hereafter referred to as Weibull models. Prediction accuracies and sensitivities based on $p^{*}$ for all ranged from 41 to $91 \%$. Model 4 had the highest sensitivity on the training data set, whereas the highest specificity was observed for model 8 . However, model 8 had the lowest sensitivity

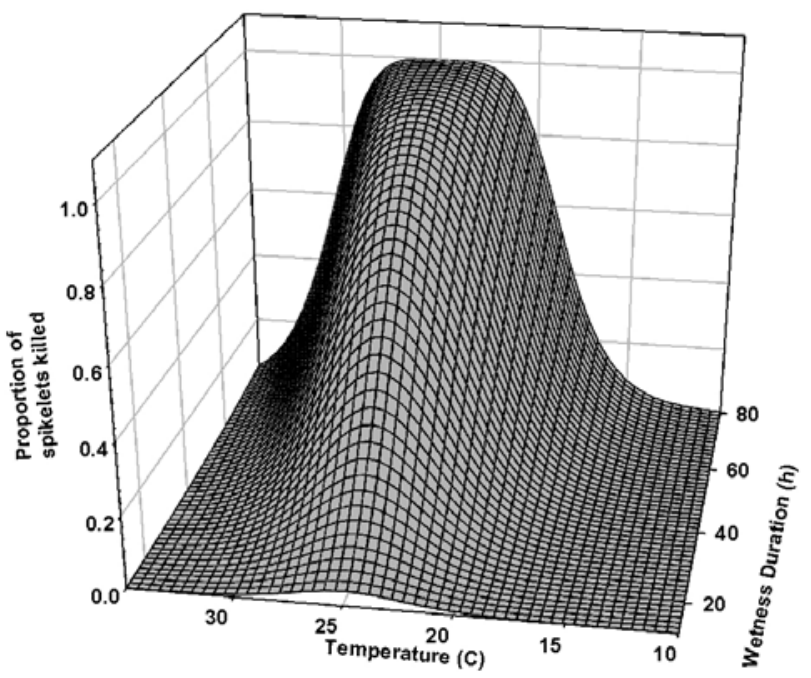

Fig. 1. Predicted response surface generated by the Weibull function using disease incidence data from a controlled environment study with wheat. Parameters $A, C, D$, $E, F, G$, and $H$ were $1.0,12.0,2.6475,0.0252,25.0,0.4804$, and 1.0744 , respectively. compared to others on the training data set. Models 3 and 6 had sensitivity and total accuracies greater than $80 \%$. The ROC curves for models 3, 4, and 6 were shown in Figure 2, and their corresponding AUROCs were similar. The thresholds for the Weibull variables to classify the event as positive or negative for eDON at corresponding $p^{*}$ were also given in Table 4.

Model validation. The validation data set had 17 events with DON concentration $\geq 0.5 \mathrm{mg} / \mathrm{kg}$ and 12 events with DON concentration $<0.5 \mathrm{mg} / \mathrm{kg}$. When models were evaluated using this data, model 4 had highest sensitivity with lowest specificity and model 8 had highest specificity with lowest sensitivity. Sensitivity was above $80 \%$ for models 1,3 , and 7; however, models 1 and 7 had specificities of only 58 and $50 \%$, respectively. Overall, model 3 had highest total prediction accuracy (79\%) and highest sensitivity $(82 \%)$ with moderate specificity $(75 \%)$.

Optimal threshold derivation. Since models 3,4 , and 6 had higher sensitivities on the training data set, these models were considered for the calculation of optimal decision threshold $\left(p_{T}\right)$ for a given level of Prev. Estimates for $k e, r e, k p$, and $r p$ of equation 5 for models 3, 4, and 6 are shown in Table 5. The estimates were significant with low standard error and provided a reasonable fit for the observed values (results not shown). The optimal decision threshold $\left(p_{T}\right)$ decreased rapidly with increasing Prev (Fig. 3). For example, when Prev is 0.5 , the value for $p_{T}$ was $0.12,0.14$, and 0.14 for models 3,4 , and 6 , respectively. In our data set, the average Prev was $0.23(34 / 150)$, and the values of $p_{T}$ at this Prev for models 3,4 , and 6 were $0.28,0.35$, and 0.34 , respectively. For model 3, the model predictions reduced the average costs compared with the cost associated with fungicide application when Prev was between 0.01 and 0.54 (Fig. 4). In contrast, models 4 and

Table 3. Pearson correlation coefficient between variables obtained from Weibull function and the disease metrics

\begin{tabular}{|c|c|c|c|c|c|c|}
\hline \multirow[b]{2}{*}{ Variable name } & \multirow[b]{2}{*}{$t$} & \multirow[b]{2}{*}{$w$} & \multicolumn{4}{|c|}{ Disease metrics $^{y}$} \\
\hline & & & Incidence & Field severity & DON & eDON \\
\hline $\mathrm{Wb}_{1}$ & AvgT & RH90 & $0.55 * * * \mathrm{z}$ & $0.37 * * *$ & $0.40 * * *$ & $0.62 * * *$ \\
\hline $\mathrm{Wb}_{2}$ & & drRH90 & $0.44 * * *$ & $0.37 * * *$ & $0.24 * *$ & $0.59 * * *$ \\
\hline $\mathrm{Wb}_{3}$ & & wRH90 & $0.57 * * *$ & $0.42 * * *$ & $0.39 * * *$ & $0.60 * * *$ \\
\hline $\mathrm{Wb}_{4}$ & AvgMinT & RH90 & $0.45^{* * *}$ & $0.27 * * *$ & $0.39 * * *$ & $0.58 * * *$ \\
\hline $\mathrm{Wb}_{5}$ & & drRH90 & $0.49 * * *$ & $0.43^{* * *} *$ & $0.29 * * *$ & $0.63 * * *$ \\
\hline $\mathrm{Wb}_{6}$ & & wRH90 & $0.47 * * *$ & $0.30 * * *$ & $0.40 * * *$ & $0.60 * * *$ \\
\hline $\mathrm{Wb}_{7}$ & AvgMaxT & RH90 & $0.36^{* * * *}$ & $0.35^{* * *} *$ & $0.22 * *$ & $0.40 * * *$ \\
\hline $\mathrm{Wb}_{8}$ & & drRH90 & $0.55^{* * *}$ & $0.71 * * *$ & $0.14^{*}$ & $0.47 * * *$ \\
\hline $\mathrm{Wb}_{9}$ & & wRH90 & $0.30 * * *$ & $0.29 * * *$ & $0.20 * *$ & $0.35 * * *$ \\
\hline
\end{tabular}

${ }^{y}$ FHB incidence: percent infected spikes; field severity: number of symptomatic spikelets/total number of spikelets; DON: mean deoxynivalenol concentration in $\mathrm{mg} / \mathrm{kg}$ obtained from replicated plots at a single location; eDON: a binary variable defined as 1 if the mean DON concentration is greater than or equal to $0.5 \mathrm{mg} / \mathrm{kg}, 0$ otherwise;

z ***: Significantly different from zero at $P<0.001$ level; **: significantly different from zero at $P<0.05$ level; $*$ significantly different from zero at $P<$ 0.1 level.

Table 4. Logistic regression equations and the accuracies in predicting eDON on both training and validation data sets

\begin{tabular}{|c|c|c|c|c|c|c|c|c|c|c|}
\hline \multirow[b]{2}{*}{$\#$} & \multirow[b]{2}{*}{$\begin{array}{l}\text { Model equation }^{w} \\
\left(\beta_{0}+\beta_{1} \times X\right)\end{array}$} & \multirow[b]{2}{*}{$\operatorname{AUROC}^{\mathbf{x}}$} & \multirow[b]{2}{*}{$p^{* x}$} & \multirow[b]{2}{*}{$\begin{array}{l}\text { Predictor variable } \\
\text { value at } p^{* y}\end{array}$} & \multicolumn{3}{|c|}{ Training data $(n=150)$} & \multicolumn{3}{|c|}{ Validation data $(n=29)$} \\
\hline & & & & & TPP $^{x}$ & TNP & $\begin{array}{c}\text { Total } \\
\text { accuracy }\end{array}$ & TPP $^{x}$ & TNP ${ }^{x}$ & $\begin{array}{c}\text { Total } \\
\text { accuracy }\end{array}$ \\
\hline 1 & $-3.34+5.39 \times \mathrm{Wb}_{1}$ & $0.89 * \mathrm{Z}$ & 0.23 & 0.40 & 76 & 85 & 83 & 82 & 58 & 72 \\
\hline 2 & $-3.20+134.34 \times \mathrm{Wb}_{2}$ & $0.88 *$ & 0.33 & 0.02 & 79 & 94 & 91 & 59 & 75 & 66 \\
\hline 3 & $-3.85+5.16 \times \mathrm{Wb}_{3}$ & $0.88 *$ & 0.28 & 0.56 & 82 & 84 & 84 & 82 & 75 & 79 \\
\hline 4 & $-2.34+37.96 \times \mathrm{Wb}_{4}$ & $0.89 *$ & 0.13 & 0.01 & 91 & 75 & 79 & 100 & 33 & 72 \\
\hline 5 & $-2.91+2410.36 \times \mathrm{Wb}_{5}$ & $0.87 *$ & 0.34 & 0.00 & 74 & 92 & 88 & 59 & 75 & 66 \\
\hline 6 & $-2.48+26.90 \times \mathrm{Wb}_{6}$ & $0.88 *$ & 0.16 & 0.03 & 85 & 79 & 81 & 71 & 50 & 62 \\
\hline 7 & $-3.91+3.86 \times \mathrm{Wb}_{7}$ & $0.80 *$ & 0.23 & 0.70 & 82 & 67 & 71 & 82 & 50 & 69 \\
\hline 8 & $-2.08+14.76 \times \mathrm{Wb}_{8}$ & $0.72 *$ & 0.46 & 0.13 & 41 & 96 & 84 & 53 & 92 & 69 \\
\hline 9 & $-3.99+3.58 \times \mathrm{Wb}_{9}$ & $0.79 *$ & 0.37 & 0.97 & 68 & 81 & 78 & 71 & 50 & 62 \\
\hline
\end{tabular}

${ }^{\mathrm{w}}$ Model equation was expressed in terms of $\operatorname{logit}(p)$, where $\operatorname{logit}(p)=\log (p /(1-p))$. That is, right side of the model equation $\operatorname{logit}(p)=\beta_{0}+\beta_{1} X$ was given

x AUROC: area under the ROC curve; $p^{*}$ : cut-off probability to classify an event as positive eDON event based on maximizing Youden's index; TPP: sensitivity; TNP: specificity.

y Value of corresponding predictor obtained from $\frac{\operatorname{logit}\left(p^{*}\right)-\beta_{0}}{\beta_{1}}$, where $p^{*}$ was from Youden's index, and estimates for $\beta_{0}$ and $\beta_{1}$ were given in the model equation.

$\mathrm{z} *$ : Significantly greater than 0.5 at $P<0.001$ level. 
6 reduced the average cost when Prev was between 0.01 to 0.38 (Fig. 4). Above these values of Prev, average costs associated with model predictions were higher than the cost associated with fungicide spray. Furthermore, the cost associated with model 3 was less than that of models 4 and 6 (Fig. 4).

\section{Discussion}

The primary purpose of this study was to evaluate the correlation between FHB and DON concentration and to develop weatherbased models to estimate the risk of economic DON accumulation in barley. A significant positive correlation was observed between FHB (incidence and field severity) and DON concentration, as was already noted by others for both barley $(4,33)$ and wheat $(28)$. Among the nine potential models developed to predict eDON, models 3,4 , and 6 were predictive of eDON risk under field conditions in the region, with high sensitivity, moderate specificity, and overall accuracy greater than $80 \%$. Further evaluation of these models suggested that model 3 would be the best choice to provide DON risk predictions to barley growers.

The measurements of temperature (AvgT, AvgMinT, and AvgMaxT) and wetness duration (RH90, drRH90, and wRH90) in the field represented situations when the environment should be optimal for infection by G. zeae and subsequent symptom development $(1,9,27)$. For example, drRH90 measured prolonged periods of high humidity, a condition that has been found to be significantly associated with FHB symptom development and DON concentration for both wheat $(1,5,6,9,19,26,27)$ and barley $(25,40)$. Similar conditions are important in many other fungal pathosystems $(12,29)$. AvgT has also been found to be another critical environmental factor influencing this pathosystem (38). Even though the variables considered in this study were similar to those presented elsewhere $(9,10,19,26)$, the approach differed in that a specific infection model was utilized, and since incidence was significantly associated with DON in barley, this then could be used for
Model 3

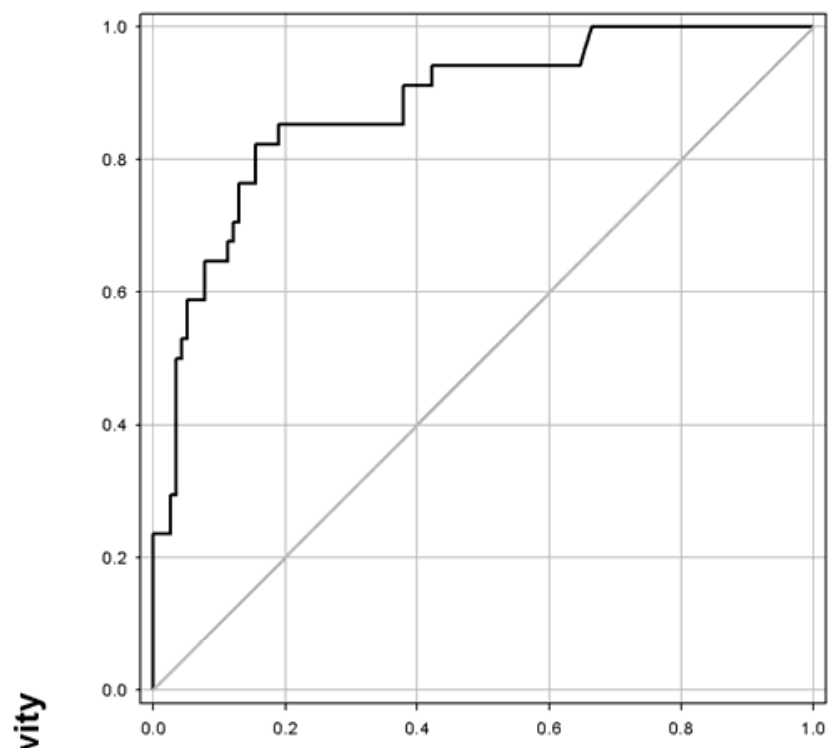

Model 6

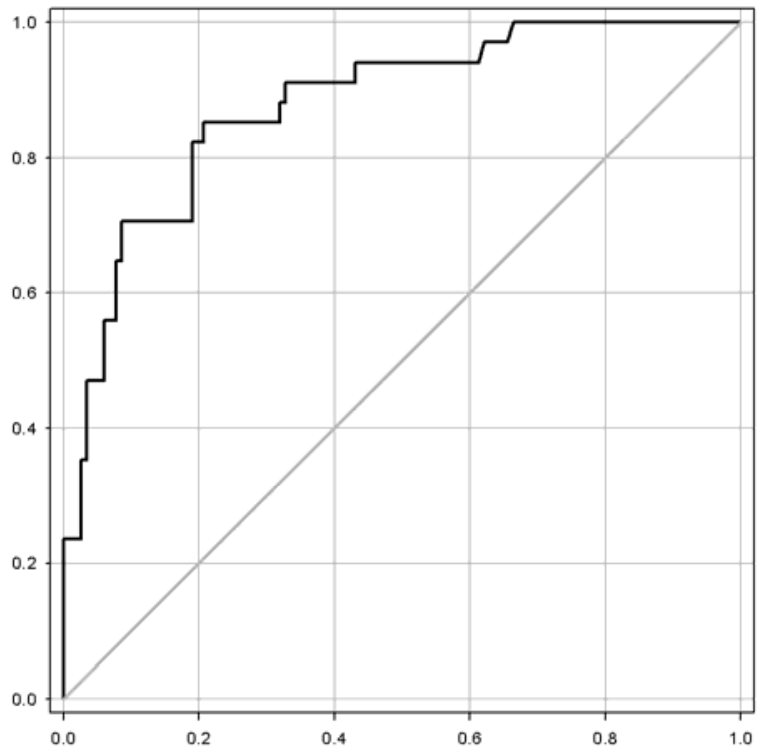

\section{Model 4}

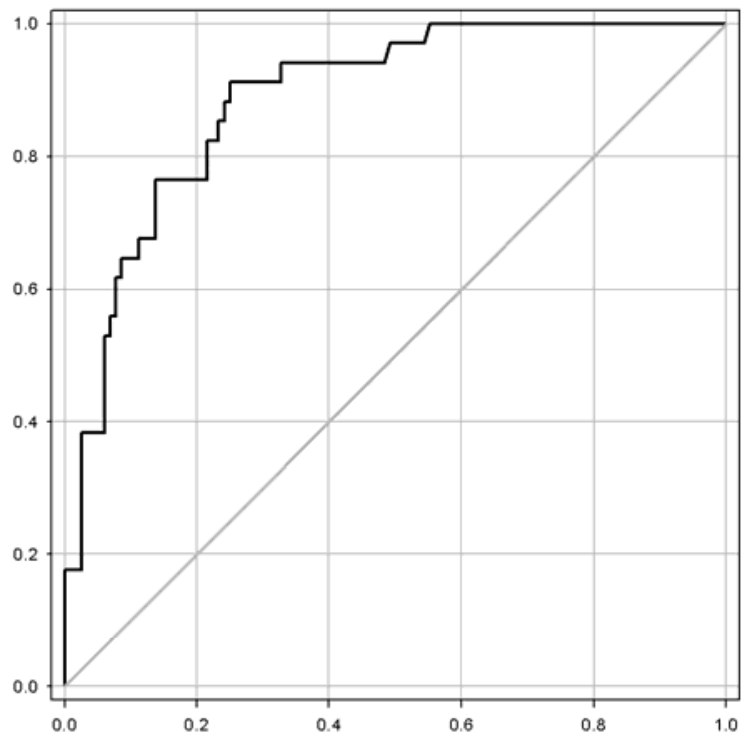

\section{1- Specificity}

Fig. 2. Receiver operating characteristic (ROC) curves for the logistic regression models 3,4 , and 6 . Sensitivity (TPP) represents the proportion of true classification of positive eDON events, and specificity (TNP) represents the proportion of true classification of negative eDON events. 
eDON prediction. The significant association found between incidence and eDON in the malting barley cultivars evaluated in this study could potentially be used in other efforts, such as breeding, since it would be faster than counting spikelets.

Among the three most accurate models, overall performance (accuracy, sensitivity, and specificity) was higher for model 3 (AvgT and wRH90) compared to the other two when evaluated using the 2010 field data. Although model 4 had the highest sensitivity for the training $(91 \%)$ and validation $(100 \%)$ data sets, it failed to predict the negative eDON events in 2010 (specificity $=33 \%$ ). The low specificity and high sensitivity for model 4 was likely due to the lower cut-off value for $\mathrm{Wb}_{4}$, which had a higher probability of classifying all the events as positive eDON events. In addition, the range of Prev in which the average cost per hectare associated with the model was lower than the routine fungicide spray was widest for model 3 (Fig. 4). This indicated that model 3 is likely to be more robust and able to accurately predict eDON using environmental conditions not present in the training data set.

Model 3 had 18 false positive (FP) and 6 false negative (FN) events on the training data set (2005-2009), and 3 FP and 3 FN events on the validation data set (2010). Among the six FN events in the training data set, two had either an intense rain event (37 $\mathrm{mm}$ ) or longer duration of humidity greater than $90 \%$ (26 h) occurring within 3 days after the heading day. This was outside the timeinterval considered in this study, but has been observed as having an impact on both disease and DON in this pathosystem $(5,9,19,38)$. The remaining FN events either had DON concentrations just slightly above the threshold (e.g., $0.65 \mathrm{mg} / \mathrm{kg}$ ) or were from the 6-row cultivars (Robust or Tradition). Among the $18 \mathrm{FP}$ events on the training data set, $50 \%$ were identified as from the 2row cultivar (Conlon). This indicated that the 2-row cultivar tested seemed to be more resistant to DON accumulation than the 6-row cultivars, which has been noted previously (4). Therefore, even though cultivar was not significant $(P=0.61)$, a model with different thresholds varying with cultivar resistance might be more accurate. Based on the results of this study, model 3 would be the most appropriate for predicting the occurrence of $\geq 0.5 \mathrm{mg} / \mathrm{kg}$ DON in an FHB-susceptible malting barley crop grown in the U.S. Northern Great Plains. This model had high predictive power on both training and validation data sets while appropriately considering the optimal environmental conditions for G. zeae. That stated, the data used in this study was limited to six growing seasons and does not necessarily represent all of the possible weather conditions that could occur in the region and influence FHB disease development or DON accumulation. Further validation is required and will be conducted during subsequent growing seasons. The use of an infection model built from wheat data might not be completely appropriate for barley; however, the similarity of the variables developed in this effort with the numerous wheat models means that there is probably little difference between infection in the two crops. It might even be possible to generate a universal infection model which could be included as a component of a more comprehensive mechanist model. This study was restricted to only three cultivars, albeit they were widely grown in the region during the testing period. The release and deployment of malting barley cultivars with increased levels of FHB resistance will undoubtedly impact model accuracy. Experiments are currently underway that should allow for the development of a cultivar resistance parameter. It should also be noted that model 3, or any model using preheading weather data, may fail when the environment becomes highly conducive after heading. This was found to occur at several of the locations during the study, resulting in false negatives. This phenomenon requires further study in barley. Current fungicide labels prohibit late-season applications due to preharvest intervals, and therefore, a model that supports a fungicide application decision process based on the most susceptible crop stage in terms of DON accumulation (i.e., heading period) is the most appropriate considering these constraints.

\section{Acknowledgments}

This material is based upon work supported by the U.S. Department of Agriculture, under Agreement No. 59-0790-4-107. This is a cooperative project with the U.S. Wheat \& Barley Scab Initiative. Any opinions, findings, conclusions, or recommendations expressed in this publication are those of the authors and do not necessarily reflect the view of the U.S. Department of Agriculture. We thank:

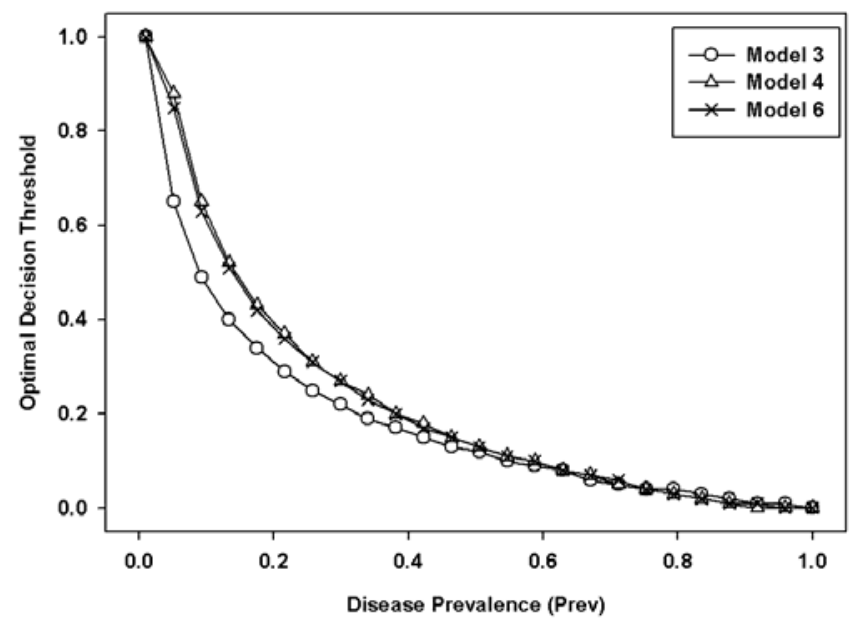

Fig. 3. Relationship between optimal decision threshold $\left(p_{T}\right)$ and disease prevalence (Prev) for the selected models 3, 4, and 6. At each Prev, $p_{T}$ was obtained by minimizing the total average cost associated with misclassified events.

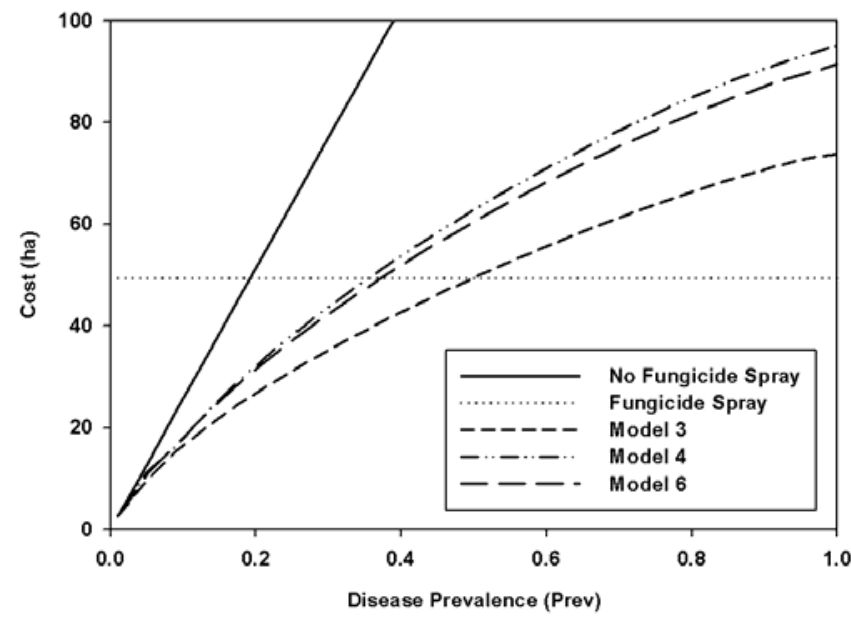

Fig. 4. Average costs per hectare associated with no recommendation of fungicide spray, recommendation of fungicide spray each season, and applications based on the risk estimated by models 3,4 , and 6 . Average cost for fungicide spray per hectare is $\$ 50$. Costs are expressed in terms of U.S. dollar per hectare.

Table 5. Parameter estimates and standard errors of the nonlinear regression models approximating the sensitivity and specificity of the models predicting positive eDON events

\begin{tabular}{llrrr}
\hline & $\boldsymbol{k e}^{\mathbf{y}}$ & $\boldsymbol{r e}$ & $\boldsymbol{k} \boldsymbol{p}$ & $\boldsymbol{r} \boldsymbol{p}$ \\
\hline Model 3 & $0.32(0.02)^{\mathrm{z}}$ & $2.34(0.08)$ & $257.20(46.45)$ & $0.19(0.01)$ \\
Model 4 & $0.60(0.04)$ & $1.52(0.08)$ & $66.06(21.54)$ & $0.26(0.02)$ \\
Model 6 & $0.55(0.03)$ & $1.59(0.07)$ & $85.18(27.18)$ & $0.24(0.02)$ \\
\hline
\end{tabular}

\footnotetext{
y Parameters were defined in text for equation 4.
}

z Parameter estimate (standard error). 
C. Nelson at SDSU, P. Gross, R. Brueggeman, and R. Horsley at NDSU, C. Motteberg at UMN-Crookston, and J. Eckhoff at Montana State University for providing technical support or guidance for this project.

\section{Literature Cited}

1. Andersen, A. L. 1948. The development of Gibberella zeae head blight of wheat. Phytopathology 38:595-611.

2. Anonymous. 2011. Know your malting barley varieties. http://www. ambainc.org/media/AMBA_PDFs/Pubs/2011_KYMBV.pdf. American Malting Barley Association Inc. Last accessed: September 15, 2011.

3. Arauz, L. F., Neufeld, K. N., Lloyd, A. L., and Ojiambo, P. S. 2010. Quantitative models for germination and infection of Pseudoperonospora cubensis in response to temperature and duration of leaf wetness. Phytopathology 100:959-967.

4. Choo, T. M., Martin, R. A., Ho, K. M., Shen, Q., Fedak, G., Savard, M., Voldeng, H., Falk, D. E., Etienne, M., and Sparry, E. 2004. Fusarium head blight and deoxynivalenol accumulation of barley in Eastern Canada: Cultivar response and correlation analysis. Plant Dis. 88:837-844.

5. Cowger, C., Patton-Ozkurt, J., Brown-Guedira, G., and Perugini, L. 2009. Post-anthesis moisture increased Fusarium head blight and deoxynivalenol levels in North Carolina winter wheat. Phytopathology 99:320-327.

6. Culler, M. D., Miller-Garvin, J. E., and Dill-Macky, R. 2007. Effect of extended irrigation and host resistance on deoxynivalenol accumulation in Fusarium-infected wheat. Plant Dis. 91:1464-1472.

7. De Wolf, E., Molineros, J., Madden, L., Lipps, P., Knight, P., and Miller, D. 2005. Future directions in the development and application of risk assessment models for Furarium head blight. Page 117 in: Proc. 2005 Natl. Fusarium Head Blight Forum, Milwaukee, WI.

8. De Wolf, E. D., and Isard, S. A. 2007. Disease cycle approach to plant disease prediction. Annu. Rev. Phytopathol. 45:203-220.

9. De Wolf, E. D., Madden, L. V., and Lipps, P. E. 2003. Risk assessment models for wheat Fusarium head blight epidemics based on within-season weather data. Phytopathology 93:428-435.

10. Detrixhe, P., Chandelier, A., Cavelier, M., Buffet, M., and Oger, R. 2003. Development of an agrometeorological model integrating leaf wetness duration estimation to assess the risk of head blight infection in wheat. Asp. Appl. Biol. 68:199-204.

11. Dill-Macky, R., and Jones, R. K. 2000. The effect of previous crop residues and tillage on Fusarium head blight of wheat. Plant Dis. 84:71-76.

12. Duthie, J. A. 1997. Models of the response of foliar parasites to the combined effects of temperature and duration of wetness. Phytopathology 87:1088-1095

13. Fabre, F., Dedryver, C. A., Leterrier, J. L., and Plantegenest, M. 2003. Aphid abundance on cereals in autumn predicts yield losses caused by Barley yellow dwarf virus. Phytopathology 93:1217-1222.

14. Faraway, J. J. 2005. Linear models with R. Chapman \& Hall/CRC, Boca Raton, FL.

15. Garbe, L.-A., Schwarz, P., and Ehmer, A. 2009. Beer gushing. Pages 185212 in: Beer: A Qualitative Perspective. C. W. Bamforth, ed. Academic Press, New York.

16. Gjertsen, P. 1967. Gusing in beer; its nature, cause and prevention. Brew. Digest 42:80-84

17. Gleason, M. L., Taylor, S. E., Loughin, T. M., and Koehler, K. J. 1994. Development and validation of an empirical model to estimate the duration of dew periods. Plant Dis. 78:1011-1016.

18. Harrell, F. E., Jr. 2001. Regression Modeling Strategies. Springer, New York.

19. Hooker, D. C., Schaafsma, A. W., and Tamburic-Ilincic, L. 2002. Using weather variables pre- and post-heading to predict deoxynivalenol content in winter wheat. Plant Dis. 86:611-619.

20. Klem, K., Vanova, M., Hajslova, J., Lancova, K., and Sehnalova, M. 2007.
A neural network model for prediction of deoxynivalenol content in wheat grain based on weather data and preceding crop. Plant Soil Environ. 53:421-429.

21. Madden, L. V. 2006. Botanical epidemiology: Some key advances and its continuing role in disease management. Eur. J. Plant Pathol. 11:3-23.

22. Markell, S. G., and Francl, L. J. 2003. Fusarium head blight inoculum: Species prevalence and Gibberella zeae spore type. Plant Dis. 87:814-820.

23. McRoberts, N., Hall, C., Madden, L. V., and Hughes, G. 2011. Perception of disease risk: From social construction of subjective judgments to rational decision making. Phytopathology 101:654-665.

24. Metz, C. E. 1978. Basic principles of ROC analysis. Nucl. Med. 8:283-298.

25. Mihuta-Grimm, L., and Foster, R. L. 1989. Scab on wheat and barley in Southern Idaho. Plant Dis. 73:769-771.

26. Moschini, R. C., and Fortugno, C. 1996. Predicting wheat head blight incidence using models based on meteorological factors in Pergamino, Argentina. Eur. J. Plant Pathol. 102:211-218.

27. Osborne, L. E., and Stein, J. M. 2007. Epidemiology of Fusarium head blight on small-grain cereals. Int. J. Food Microbiol. 119:103-108.

28. Paul, P. A., Lipps, P. E., and Madden, L. V. 2006. Meta-analysis of regression coefficients for the relationship between Fusarium head blight and deoxynivalenol content of wheat. Phytopathology 96:951-961.

29. Pfender, W. F. 2003. Prediction of stem rust infection favorability, by means of degree-hour wetness duration, for perennial ryegrass seed crops Phytopathology 93:467-477.

30. R Development Core Team. 2009. R: A language and environment for statistical computing. R Foundation for Statistical Computing, Vienna.

31. Rao, P. S., Gillespie, T. J., and Schaafsma, A. W. 1998. Estimating wetness duration on maize ears from meteorological observations. Can. J. Soil Sci. 78:149-154.

32. Rossi, V., Giosue, S., Pattori, E., Spanna, F., and Del Vecchio, A. 2003. A model estimating the risk of Fusarium head blight on wheat. OEPP/EPPO Bull. 33:421-425

33. Salas, B., Steffenson, B. J., Casper, H. H., Tacke, B., Prom, L. K., Fetch, T. G., Jr., and Schwarz, P. B. 1999. Fusarium species pathogenic to barley and their associate mycotoxins. Plant Dis. 83:667-674.

34. Sarlin, T., Laitila, A., Pekkarinen, A., and Haikara, A. 2005. Effects of three Fusarium species on the quality of barley and malt. J. Am. Soc. Brew. Chem. 63:43-49.

35. Schmale, D. G., Leslie, J. F., Zeller, K. A., Saleh, A. A., Shields, E. J., and Bergstrom, G. C. 2006. Genetic structure of atmospheric populations of Gibberella zeae. Phytopathology 96:1021-1026.

36. Stein, J. M., Osborne, L. E., Bondalapati, K. D., Glover, K. D., and Nelson, C. A. 2009. Fusarium head blight severity and deoxynivalenol concentration in wheat in response to Gibberella zeae inoculum concentration. Phytopathology 99:759-764.

37. Sutton, J. C., Gillespie, T. J., and Hilderbrand, P. D. 1984. Monitoring weather factors in relation to plant disease. Plant Dis. 68:78-84.

38. Van Der Fels-Klerx, H. J., Burgers, S. L. G. E., and Booij, C. J. H. 2010. Descriptive modelling to predict deoxynivalenol in winter wheat in the Netherlands. Food Addit. Contam. A. 27:636-643.

39. Vanova, M., Klem, K., Matusinsky, P., and Trnka, M. 2009. Prediction model for deoxynivalenol in wheat grain based on weather conditions. Plant Prot. Sci. 45:S33-S37.

40. Vestal, E. F. 1964. Barley scab in South Korea in 1963 and 1964. Plant Dis. Rep. 48:754-755.

41. Yoshida, M., Nakajima, T., Arai, M., Suzuki, F., and Tomimura, K. 2008. Effect of the timing of fungicide application on Fusarium head blight and mycotoxin accumulation in closed-flowering barley. Plant Dis. 92:1164-1170.

42. Youden, W. J. 1950. Index for rating diagnositc tests. Cancer 3:32-25.

43. Zadoks, J. C., Chang, T. T., and Konzak, C. F. 1974. A decimal code for the growth stages of cereals. Weed Res. 14:415-421. 